\title{
A WEB BASED EXPERT SYSTEM FOR MILCH COW DISEASE DIAGNOSIS SYSTEM IN CHINA
}

\author{
Libin Rong ${ }^{1,2}$ Daoliang Li ${ }^{1,2, *}$ \\ ${ }^{1}$ China Agricultural University, College of Information and Electrical Engineering, Beijing \\ 100083, China \\ ${ }^{2}$ Key Laboratory of Modern Agriculture System Integration, China Agricultural University, \\ Beijing 100083, China \\ *Corresponding Author, Tel: +86-10-62736717, Fax: +86-10-62737679, Email: \\ dliangl@cau.edu.cn or
}

Abstract: A web-based multi-models expert system called DCDDS is presented in this paper, which developed for diagnosis of dairy cow diseases through the symptoms submitted by users on web. As it is accepted that the inference engine and the relevant knowledge representation are the crucial part of diagnosis expert system, which limits its application and popularization in animal disease diagnosis. To break the limit and raise accuracy, this paper compares and appraises the existed systems and presents a solution that contains three models - Case-based reasoning (CBR), Subjective Bayesian theory and D-S evidential theory. Accordingly a knowledge representation method which can support the three different models is also designed. Up to the complicacy of the group of symptoms users acquired, they can choose which of the three models should be adopted to meet the best resolve. The performance of the proposed system was evaluated by an application to the field of dairy cow disease diagnosis using a real example of dairy cow diseases. The result indicates that the new methods have improved the inference procedures of the expert systems, and have showed that the new architecture has some advantage over the conventional architectures of expert systems on both efficiency and accuracy.

Keywords: Dairy cow disease diagnosis; Web-based expert system; Case-based reasoning; Subjective Bayesian theory; D-S evidential theory; China

Rong, L. and Li, D., 2008, in IFIP International Federation for Information Processing, Volume 259; Computer and Computing Technologies in Agriculture, Vol. 2; Daoliang Li; (Boston: Springer), pp. 1441-1445. 


\section{INTRODUCTION}

Dairy cow disease diagnosis is rather a complicated process in stockbreeding production activity because the disease commonly resulted from many conditions difficult to detect contains nutritional and environmental problems, microbe conditions and incorrect method of milking, etc. The diseases diagnosis is rather a difficult task for dairy farmers, which can take a cow breeding worker or a dairyman more than three years to be able to correctly identify diseases by self-learning and practicing. So it needs the specialist to deal with. But the experts do not often on the port for most of the culture sites are remote from expert centers which are often seated in big cities. The serious confliction of the need of dairy cow disease expertise and the fact that the supply fall short of demand present a practical need to develop a computer-based system with the ability of providing accurate, timely and remote service of disease diagnosis.

A web-based expert system for dairy cow disease diagnosis has been developed by Key Laboratory of Modern Agriculture System Integration at China Agricultural University. It was a major outcome of Huo Yingdong Young Teacher's Foundation (Contract No. 94032). This web-based intelligent system can mimic human cow disease expertise and diagnose 67 types of common dairy cow diseases with a user-friendly interface. It contains a large amount of cow disease data and images, which are used to conduct online disease diagnosis.

Experiences with developing web-based expert systems, lessons learnt and test evaluation are discussed and conclusions are provided at the end of the paper.

\section{DOMAIN DESCRIPTION}

\subsection{The knowledge of dairy cow disease}

Profitable milk production relies upon a flock of healthy, productive cows. But failures of cattle diseases diagnosis are one of the important reasons for considerable losses of income in dairy farming. Many factors can be related to dairy cow diseases and the main impact elements contain the follows:

$\diamond$ The microbe factor which contains infective pathogen and environmental pathogen.

$\diamond \quad$ The environmental factor that indicates improper management and bad sanitation, not to clean or disinfect the cowshed for example. 
$\diamond$ The unscientific milk technology which is the most important reason for mastitis.

$\diamond$ The management of feeding which is to say the cow should be fed timely and the feedstuff should be nutritious.

$\diamond \quad$ The individual factor.

\subsection{User's needs for expert system}

China is a large country and most dairy farms are spread in the remote rural areas. Although dairy farming is growing rapidly, the level of farmers' skills and knowledge are evidently low or even non-existent. As a result, cattle diseases occur frequently and the consequences on farmers' financial status are enormous, as sometimes the whole herd of cows could be infected at one time. The demand for help from experts is increasing rapidly. However, experts are scarce and not readily available, especially in rural areas. It takes long time to train novices and even longer for them to establish their experience in practice. An expert system is considered as an effective tool to help dairyman in solving the problems they meet in practice. Therefore, an expert system for dairy cow disease diagnosis is required to substitute human vets in helping farmers in China. Also the specialists need an expert system because the human expert' life is limited; he/she can only obtain limited experience. Furthermore, various personal characteristic and body or mood factors can impact their diagnosis. The computer systems have no such problems. They can accumulate knowledge all the while and always conclude the same result from the same evidences.

\section{SYSTEM DESIGN AND DEVELOPMENT}

\subsection{System architecture}

Based on the analysis and assessment of the users' needs, a web-based decision expert system called DCDDS was developed. The system is a typical three-tier web application which uses Internet Information Server (IIS), Microsoft SQL Server 2000, Windows XP as the operating system and Windows XP with Internet Explorer (IE) on the client side. The web pages were programmed using html and C\# code running from ASP.NET filter which is a component of IIS. Users (patient or medical worker) enter the system via the web. 


\subsection{Inference engine}

An inference engine of multi-models for the system is designed, which is the brain of the system. As the former described, clinical diagnosis is often complicated because similar clinical signs may arise in animals with different diseases while the same disease may well express itself in different ways in different individuals within an infected population. Hence, the diagnosis of disease is not well suited to the binary logic seen in the structure of many rule-based algorithms, which is often adopted in the lots of diagnosis systems. The adoption of three algorithms includes CBR, subjective Bayesian theory and D-S evidential theory provides a moreflexible method for use in disease diagnosis. Because the different three algorithms can get results with different efficiency and exactness, users can choose one of the three models in specifically occasion to get a reliable diagnosis result. The CBR fit for familiar symptom groups and it has high efficiency of runtime. The subjective Bayesian theory can be adapted with the groups of less and inconspicuous symptoms. It can deduce the possible disease and figure out its probability. However, when the information volume is very low and the use of probability functions is not possible, it is advisable to use other alternative techniques. In this case, a candidate for managing uncertainty in the expert systems is the Dempster-Shafer evidence theory.

\subsection{Flowchart}

The DCDDS is composed of four subsystems: case management, diagnosis, prevention and cure and medicine management. Users can choose to browse the records about their cows when login in the system. They can also choose to study by looking through the medicine component and the prevention component. Certainly, the main function of the system is to diagnose. If users choose to diagnose, they will be shown the first step to submit the environment information around the sick cow and to select the symptoms visible. Within the set of symptoms the users selected, the system will look for relevant disease. Only when some diseases can explain and match as many symptoms as possible based on the rule, the process is finished. To do this, the system will first look for a disease that can explain all the symptoms. If there is no such disease, it will look for a disease that can explain all but one symptoms, etc., until it finds a possible disease [2]. The users also can select the disease the users supposed based on his experience. With the set of disease the user selected, the system will look for relevant symptoms and output them to let user confirm. When get a disease finally, a proposal about prevention and cure will be given. At the end, the 
users will be asked if to save the diagnosis result as a case history to be checked in the future.

\section{DISCUSSIONS AND CONCLUSIONS}

This system has the following characteristics:

It is a web-based expert system that provides an easy access for dairymen. Comparing with most of the exist expert systems which based on $\mathrm{C} / \mathrm{S}$ mode or setup on a stand alone computer, the DCDDS is easy to upgrade the program. With its online data and knowledge acquisition system, farmers, technicians and experts can easily update the knowledge base and data base with new instance. Users can reach the system only with a PC connected with the internet and they can get help from the expert system with up-tothe-minute version.

The users' feedback form serves as an effective and efficient mechanism to collect specialists and common users' comments, problems and suggestions.

It also provides general information support tools, such as a dictionary, information on medicine, contact information of medicine vendors, etc, which help the users to study online.

\section{ACKNOWLEDGEMENT}

This program is supported by Huo Yingdong Young Teacher's Foundation (Contract No. 94032). We also would like to thank many domain experts from China Agriculture University.

\section{REFERENCE}

Schreiber, G., Wielinga, B., de Hoog, R., Akkermans, H., van de Velde, W., CommonKADS: a comprehensive methodology for KBS development. IEEE Expert 12, 2837 (1994).

Kramers, M.A., Conijn, C.G.M., Bastiaansen, C. EXSYS, an Expert System for Diagnosing Flowerbulb Diseases, Pests and Non-parasitic Disorders. Agricultural Systems Volume: 58, Issue: 1, September, 1998, pp. 57-85. 